\title{
Studies on Garden Path Phenomenon in English
}

\section{Rui Luo ${ }^{\text {a }}$}

\begin{abstract}
Garden path phenomenon is a term that originated from psycholinguistic field. As a special temporary or local ambiguity in language processing, it has been widely explored and studied from aspects of semantics, syntax, pragmatics as well as psycholinguistics and cognitive linguistics over the years, since it was first put forward by Bever in the 1970s. The research of garden path phenomenon is of considerable significance both theoretically and practically. This paper is designed to explore the garden path phenomenon in English through literature and examples. Some basic concepts and main triggering factors of garden path phenomenon are analyzed. This paper explores the triggering factors of garden path phenomenon from the aspects of syntax, semantics, and pragmatics. The major finding is that garden path phenomenon can help learners understand the operation of human language processing mechanism and improve their abilities to deal with garden path sentences, which is of great benefits to learn and understand English language.
\end{abstract}

\section{Keywords}

Garden path phenomenon, ambiguity, triggering factors

Garden path phenomenon is a kind of temporary or local ambiguity in the parsing process of language. To be more specific, when comprehending garden path sentence, people tend to misinterpret the sentence at the very start and finally have to go back to comprehend it and get another new interpretation. Since this special temporary ambiguity phenomenon was first proposed by psycholinguist Bever (1970), it has been the hot topic among linguists and psychologists for ages. By now we can divide the studies of garden path phenomenon into three stages according to emphases on the research. From 1970s to 1980s, researches were conducted from the aspects of psychology and psycholinguistics. Researchers put forward various sentence parsing models to explain this phenomenon. Then since the late 1980s, scholars started to explore the production of garden path phenomenon based on grammatical analysis. At that time, it was believed that garden path effect was caused because interpreters offend against some syntactic rules. Recently, researchers have begun to illustrate garden path phenomenon by comprehensive strategies. Those previous studies have made a lot of contributions to the later deeper studies. This paper illustrates some basic concepts and triggering factors of garden path phenomenon, and tries to expound the enlightenment of garden path phenomenon on interpreting humorous words and conundrums, so as to help learners have a good knowledge of the operation of human language processing mechanism.

\section{aZhoukou Normal University, China}

\section{Correspondent Author:}

Rui Luo, School of Foreign Languages, Zhoukou Normal University, Zhoukou, Henan Province, China, post code: 466001 


\section{GARDEN PATH PHENOMENON}

\section{Definition of Garden Path Phenomenon}

Garden path phenomenon is a special temporary or local ambiguity in language processing. As an interesting language phenomenon, garden path phenomenon has been inevitably explored and defined by many scholars from different aspects based on their research emphasis.

In 1970, psycholinguist Bever firstly put forward garden path phenomenon and defined that: "A sentence is first comprehended in the conventional way until it is only later discovered that the first understanding is incorrect. Then on the purpose of re-understanding, non-conventional means are used to reprocess the input language by going back to the bifurcation” (Bever 1970: 279). It is defined from the aspect of psycholinguistics, which clearly shows us the human language processing mechanism.

There is another definition from psycholinguistic perspective:

The general tendency for all listeners and readers to make increasingly confident predictions about the meaning of a sentence as it progresses is well-attested in psycholinguistics and is colorfully called garden-pathing. One well-documented example of this phenomenon is the way comprehension is temporarily impeded when the listener or reader meanders down the wrong garden path in comprehending a string of words. (Scovel 1998: 65)

According to this definition, it seems that we construct syntactic representations usually from the beginning of the sentences to the end. Therefore, when we listen or read a word, we will think about its influence on the meaning of the following information and before reading the following information, we will make a number of assumptions about how the sentence will proceed. It should be no wonder that we misinterpret garden path sentence due to these assumptions in our minds. In fact, it is believed that garden path phenomenon is less common in spoken communication than in written text because of the stress, tone, and intonation of speech.

Ferreira defined garden path as "syntactically challenging but essentially unambiguous sentences” (Ferreira 2003: 164), which indicates that garden path sentences are not truly ambiguous but difficult to interpret due to some syntactic factors. However, Ferreira's definition seems to be too narrow because there are more than syntactic factors but many other whatever factors to lead to this temporary ambiguity.

According to the different definitions above, we can find an interesting feature of garden path phenomenon: It is often the case that the interpretation that people are reluctant to accept is correct in garden path sentence.

\section{The Distinction Between Permanent Ambiguity and Temporary Ambiguity}

There are two kinds of ambiguous sentences including permanent ambiguity and temporary ambiguity, or, global ambiguity and local ambiguity. Permanent ambiguity or global ambiguity is a special phenomenon in language, in which, the form or the structure is grammatically accepted but its content has two or more meanings or illustrations. Garden path phenomenon is the temporary ambiguity which could be clearly illustrated by the following examples:

(1) Jack saw his mother with a telescope;

(2) The girl read the article smiled.

Sentence (1) is a permanent ambiguity which can be comprehended that Jack used a telescope to see his mother or that Jack saw his mother holding a telescope. In contrast, sentence (2) is a temporary ambiguity but not true ambiguity. When comprehending sentence (2), at first we have a strong tendency to read it as "the girl read the article". But when we get to the word "smiled", we are confused because the sentence has no room for an extra VP (Verb Phrase). So we reconstruct the sentence to make "smiled" main verb then reread it as the girl who read 
the article smiled. Through the above two sentences, it could be concluded that garden path phenomenon is temporary ambiguity. Only we distinguish garden path phenomenon from permanent ambiguity can we explore this phenomenon well.

\section{TRIGGERING FACTORS OF GARDEN PATH PHENOMENON}

\section{Syntactic Factors}

The garden path phenomenon in syntax is mostly caused by the complexity of the sentence. There are mainly the following three triggering factors.

Firstly, the guide word is omitted in complex sentence. This type of garden path phenomenon mainly occurs in object clause and attributive clause.

Let us see the following examples:

(1) She told her daughter a dream could be achieved;

(2) The old man told the story complained to his son.

In sentence (1), the guide word of this object clause is omitted. Thus, at first people will regard the noun phrase "a dream" as the object of a simple sentence, but later will realize that in fact "a dream" is the subject of the object clause, which triggers garden path phenomenon. Sentence (2) is an attributive clause, which omits the relative word "who" and verb "was". So when readers do not get to the verb "complained", they are inclined to comprehend the sentence as the simple SVO (Subject-Verb-Object) structure. Until they read "complained", they are aware of this misinterpretation.

As for the above examples, if the omitted components are inserted back, there will be no temporary garden path ambiguity.

She told her daughter (that) a dream could be achieved.

The old man (who was) told the story complained to his son.
Secondly, in a complete sentence, other longer sentence components are inserted. We use an example to explain it:

(3) The doctor told the patient that he has trouble with to leave (Yang 2013: 77).

In this sentence, "the doctor told the patient to leave" is the complete structure of the main clause to which an attributive clause is inserted to modify the antecedent "the patient". Therefore, it causes difficulties to understand the whole sentence, namely garden path phenomenon occurs.

Thirdly, there are various possible combinations of words.

Please see the following examples:

Without her efforts would be in vain.

In fact, this sentence may not be garden path sentence for listeners because of the speakers' appropriate stops, stress, tone, and intonation. But for the non-native speakers of English, the interpretation will be a little confused and difficult. This kind of temporary ambiguity can be canceled by adding a comma to the stop of the sentence.

(4) Without her, efforts would be in vain.

\section{Semantic Factors}

It is believed that the semantic relations of internal components of sentences are important triggering factors of garden path phenomenon. There are mainly two causes in semantics.

The first factor is the default of semantic case role. Sentence comprehension is based on language perception and mental lexicon. Mental lexicon is the reality reflection on the attitude towards world and culture, which plays a great role in the assignment of the semantic case role. Semantic case role is the vocabulary that people will take for granted, which leads to garden path phenomenon.

Please see the following examples:

(1) The performer sent the flowers was very pleased (Wang 2013: 94); 
(2) The florist sent the flowers was very pleased (Wang 2013: 94).

The occurring rate of garden path sentence in sentence (2) is much higher than sentence (1). Because it is always believed that "florist" is more likely to be treated as the agent of flowers' sending than "performer". On the contrary, "performer" is more likely to be treated as the object of flowers' sending than "florist".

The second factor is the influence of polysemous words. In English, there are many polysemes, i.e. one word with different meanings and speeches. For a polyseme, the meaning more frequently used is much easier recognized and accepted. Therefore, when people comprehend sentence, they tend to choose the common meanings. But when the right meaning is inconsistent with the common meanings, garden path phenomenon will occur. Let us see the following example:

(3) The old coach the tennis team.

When reading sentence (3), most people will regard "the old coach" as the common noun phrase. But in this sentence "coach" is not the noun (someone who trains a person or team in a sport), but is the transitive verb (to teach a person or team the skills they need for a sport). Therefore, sentence (3) is likely to be garden path sentence.

\section{Pragmatic Factors}

The pragmatic factors of garden path phenomenon are explored mainly from the perspective of Relevance Theory proposed by Dan Sperber and Deirdre Wilson. According to Relevance Theory, that people comprehend utterances is on the premise of the cognitive presumption and follows the principle of relevance defined as "every act of ostensive communication communicates the presumption of its own optimal relevance" (Sperber and Wilson 1996: 158). The so-called optimal relevance is that adequate contextual effects can be obtained after making appropriate efforts to comprehend utterances.
One of the basic principles of human behavior is labor-saving, namely principle of least effort. How do people use the principle of relevance to interpret garden path phenomenon? We use an example to explain it:

(1) I saw that gasoline can explode. And a brand new gasoline can it was too (Jiang and Yin 2016: 120).

There are two kinds of interpretation of the former part of the sentence (1):

(a) I saw that it is possible for gasoline to explode;

(b) I saw that can of gasoline explode.

In general, people are inclined to interpret the former part of sentence (1) as (a), but after the sentence "And a brand new gasoline can it was too" occurs to be its context, readers have to go back to reread it and find (b) is right. If we just explore the parsing process of the former part of the sentence (1) alone, we find that when readers get to "I saw that...", there will be two interpretations of "that": a guide word of object clause or a demonstrative pronoun to modify the following noun. But because the form of demonstrative pronoun needs special context to support and this special context later occurs, it takes more efforts to the second interpretation. In contrast, for the first interpretation, the form of the guide word of object clause does not need special context to support thus does not take more efforts, which gives the priority to the first interpretation and leads to temporary garden path ambiguity.

The paper explores the triggering factors of garden path phenomenon from the aspects of syntax, semantics, and pragmatics. However, it is not said that garden path phenomenon is only caused by one certain factor and too many garden path sentences can be studied and explained from different aspects at the same time. No matter how we explain this phenomenon, there is only a conclusion that garden path phenomenon is a kind of temporary ambiguity and the whole sentence is definitely not truly ambiguous. 


\section{CONCLUSIONS}

Garden path phenomenon is a complex but interesting language phenomenon which adds difficulty to people's comprehension. To be more specific, when interpreting garden path sentence, people are inclined to misinterpret the sentence at the very start and finally have to go back to comprehend it and get another new interpretation.

Some basic concepts of garden path phenomenon are illustrated in the paper, including the definition and its distinction from permanent ambiguity. Then the triggering factors of garden path phenomenon are studied from the aspects of syntax, semantics, and pragmatics. However, it is not said that garden path phenomenon is only caused by one certain factor. In fact, many garden path sentences can be studied and explained from different aspects at the same time.

The research of garden path phenomenon is of considerable significance both theoretically and practically. Through the exploration of how this sort of sentences is mistakenly parsed, learners can have a good knowledge of the operation of human language processing mechanism. It will also help English learners to improve their abilities to deal with garden path sentences, which is of great benefits to English language learning and understanding.

\section{References}

Bever, T. 1970. Cognitive Basis for Linguistic Structures. New York: Wiley Press.

Christianson, K. 2008. "Sensitivity to Syntactic Changes in Garden Path Sentences.” Journal of Psycholinguistic Research 37(6):391-403.

Ferreira, F. 2003. "The Misinterpretation of Noncanonical Sentences.” Cognitive Psychology 47(2):164-203.

Jiang, D. J. and H. S. Yin. 2016. "Triggering Factors of 'Garden Path' Effects in English.” Journal of Qingdao University of Science and Technology 22:117-120.

Lau, E. F. and F. Ferreira. 2005. "Lingering Effects of Disfluent Material on Comprehension of Garden Path Sentences." Language and Cognitive Processes 20(5):633-666.

Niu, B. Y. 2011. Introduction to Cognitive Grammars. Shanghai: Shanghai Foreign Language Education Press.

Scovel, T. 1998. Psycholinguistics. Oxford: Oxford University Press.

Sperber, D. and D. Wilson. 1996. Relevance: Communication and Cognition. Oxford: Wiley-Blackwell Press.

Wang, L. H. 2013. "Explorations on the Triggering Factors of Garden Path Phenomenon.” Journal of Southwest Agricultural University 11:93-95.

Yang, T. Y. 2013. "An Analysis of Garden Path Phenomenon in English.” China Education Innovation Herald 29:77-79.

\section{Bio}

Rui Luo, master's degree, lecturer, School of Foreign Languages, Zhoukou Normal University, China; research fields: English linguistics and English teaching studies. 